Aim of the study: Increased Raf-1 expression has been associated with an aggressive behaviour in some carcinomas such as pulmonary carcinoma and renal carcinoma. However, its role in breast cancer, especially in basal-like carcinoma of the breast (BLBC), has not been defined.

Material and methods: The current study attempted to investigate the expression pattern of Raf-1 protein in $B L B C$, in relation to the biological behaviour and prognosis of the carcinoma. Expression of Raf-1 was detected by immunohistochemistry in carcinoma specimens from 74 cases of BLBC, and associations between their expression and the clinicopathological characteristics were statistically assessed.

Results: The patients' age, tumour size, BRCA1, and $\mathrm{p} 53$ protein expression was not significantly different between the Raf-1-positive and Raf-1-negative expression groups $(p>0.05)$. The proportion of histological grade 3 tumours was not significantly higher in the Raf-1 positive group than that of grade 2 tumours $(p>0.05)$. However, positive cytoplasmic Raf-1 expression was positively correlated to Ki-67 expression $(p<0.05)$. Also, increased Raf-1 protein was found to exert an unfavourable impact on patients' axillary lymph node metastasis and overall survival $(p<0.05)$.

Conclusions: The study implies that positive Raf-1 expression in BLBC is associated with a more aggressive phenotype and could be considered as a new prognostic biomarker for poor survival in BLBC patients.

Key words: Raf-1, basal-like breast carcinoma, aggressive phenotype.

Contemp Oncol (Pozn) 2014; 18 (6): 391-395 DOI: $10.5114 /$ wo.2014.47037

\section{Overexpression of Raf-1 in basal-like carcinoma of the breast: correlation with clinicopathology and prognosis}

\author{
Ping $\mathrm{Wu}^{1}$, Xizhou $\mathrm{Li}^{2}$, Yangmei $\mathrm{Wu}^{2}$, Wei $\mathrm{Hu}^{2}$, Yang Wang ${ }^{3}$, Li Gao ${ }^{3}$, \\ Zhongzhong Chen ${ }^{3}$, Weiqiang Zheng ${ }^{3}$
}

\begin{abstract}
'Department of Pathology, Huaian Maternity and Child Health Hospital, Huaian, Jiangsu Province, China

2Department of General Surgery, Changhai Hospital, Second Military Medical University, Shanghai, China

${ }^{3}$ Department of Pathology, Changhai Hospital, Second Military Medical University, Shanghai, China
\end{abstract}

\section{Introduction}

Breast carcinoma is a heterogeneous disease that displays a range of phenotypes with different clinical characteristics including altered clinical outcome, varying prognostic characteristics, and differential response to treatment. The clinical management of breast cancer currently still relies on traditional prognostic and predictive factors including clinical, histological, and some well-defined biological factors such as hormone receptors (estrogen receptors - ER, progesterone receptors - PR) and human epidermal growth factor receptor 2 (HER-2) expression. Cellular and molecular heterogeneity of breast carcinoma results in different distinct groups of tumours with different clinical behaviour and prognosis. Gene expression profiling of breast carcinoma has delineated five molecular subtypes based on similarities in gene expression: luminal A, luminal B, HER2 overexpressing, normal-like, and basal-like type [1]. Basal-like carcinoma of the breast (BLBC) lacks oestrogen receptor, progesterone receptor, and HER2 expression. Specific features include high proliferative rate, rapid growth, early recurrence, and decreased overall survival. Basal-like carcinoma of the breast is associated with ductal carcinoma in situ, BRCA1 mutation, brain and lung metastasis, and negative axillary lymph nodes. There are currently no specific therapies for BLBC. A number of biomarkers have emerged, which show an association with clinical outcome in basal-like or triple-negative disease, including solute carrier organic anion transporter family member 1B1 (OATP2) and fatty acid-binding protein 7 (FABP7) [2], and forkhead box protein C1 (FOXC1) [3] in basal-like cancers. Despite the overall association of these variables with prognosis and outcome, these markers are limited in their ability to capture the nuances of the complex cascade of events that drive the clinical behaviour of breast cancer [4-6].

Raf was initially discovered as a murine sarcoma and an avian carcinoma virus. As the linker between Ras (the most frequently mutated oncogene in human cancers) and the mitogen-activated protein kinase/extracellular signal-regulated kinase (MEK/ERK) module, Raf has been conclusively established as a major player in tumour development [7]. In response to growth factor stimulation and Ras activation, C-Raf (Raf-1) and B-Raf form heterodimers to yield higher MEK kinase activity for cell proliferation and tumour development [8]. Overexpression of Raf-1 has been reported in squamous cell carcinoma and lung adenocarcinoma and correlates with metastatic progression [9]. In the current study we investigated Raf-1 expression in BLBC through the immunohistochemical method and its correlation with Ki-67, p53, and other conventional clinicopathological parameters, as well as the clinical outcome of the patients. 


\section{Material and methods}

\section{Clinical data}

The breast samples of female patients consisted of 424 invasive ductal carcinomas and were retrieved from the archives of the Departments of Pathology in Huaian Maternity and Infant Health Institute and Changhai Hospital. None of the patients had received any treatment before the surgical operation. Portions of tumour were trimmed free of fat, and the axillary lymph node metastases analysed were surgically removed at the same time as the primary tumour. Formalin-fixed, paraffin-embedded histological sections of the lesions, stained with haematoxylin and eosin, were reviewed by two pathologists for designation into the above diagnostic categories. The carcinomas were evaluated for histological grade according to the modified Bloom and Richardson method.

\section{Immunohistochemistry and evaluation}

Tissue samples were fixed by immersion in buffered formalin and embedded in paraffin. Each patient with invasive breast cancer had a complete medical record. According to the Clinical Guidelines, each sample was subjected to EnVision immunohistochemistry (IHC) for ER, PR, and BRCA1 (rabbit monoclonal antibodies; Gene Corp, Shanghai, China), HER-2 (rabbit polyclonal antibodies; Dako, Glstrup China) and Raf-1 (rabbit polyclonal antibodies; Santa Cruz Biotechnology, Santa Cruz, CA, USA). The sections were counterstained with haematoxylin and coverslipped. A negative control reaction with no primary antibody was performed alongside the reaction-containing sample. Immunostaining was evaluated without knowledge of the clinical and pathological parameters. Positivity was defined as staining of $\geq 10 \%$ of nuclei in the invasive component of the tumour. For HER-2 expression, staining intensity was divided into four grades, with grades 0 and 1 considered as negative, grade 2 as indeterminate, and grade 3 as positive. Fluorescence in situ hybridisation (FISH) was performed on all grade 2 samples. Samples with $<2.2$-fold-change in expression were regarded as negative, and those with $>2.2$-fold increase were regarded

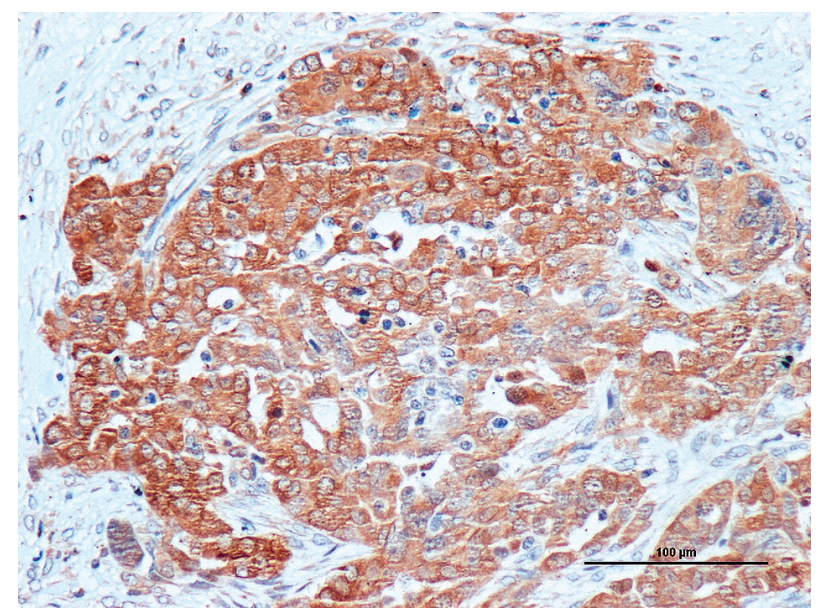

Fig. 1. Positive Raf-1 expression was located mainly in the cytoplasm of tumour cells of BLBC by immunohistochemical staining (haematoxylin counterstain) as positive for gene amplification [10]. In order to select the BLBC cases, the samples were also subjected to immunohistochemistry for CK5/6 and epidermal growth factor receptor/HER1 (EGFR, rabbit polyclonal antibodies; Santa Cruz Biotechnology, CA, USA). The BLBC subtype was selected according to the negative expression of ER \& PR and HER2, along with expression of one or a combination of the basal markers (CK5/6, CK14, vimentin, or EGFR) [11, 12].

Each patient provided informed consent prior to study participation. The study protocol was carried out with approval by the Ethics Committee of Changhai Hospital.

A total of 74 cases of carcinoma were attributed to the $B L B C$ group. The patients were followed up for at least five years, and clinical and pathological factors were recorded for analysis. Among the 74 patients, 70 cases were satisfied for the follow-up record.

\section{Statistical analysis}

The personal $\chi^{2}$ test was used to examine the statistically significant differences between expression of Raf-1 and other known predictive markers (tumour size, node involvement, histologic grade, ER/PR status, HER-2 status, p53, and Ki67). Kaplan-Meier survival curves with log-rank testing were used to assess the disease-free survival (DFS). The relative risk (RR) of reducing DFS was determined by Cox proportional hazard regression with multivariate analyses. A $P$ value of less than 0.05 was considered statistically significant.

\section{Results}

\section{Raf-1 expression in BLBC}

In the current immunohistochemical analysis of 74 BLBC cases, the tumour cells showed cytoplasmic staining for Raf1 protein (Fig. 1). No specific immunoreactivity was detected in these negative control sections. Positive expression of Raf-1 proteins was observed in 46 cases of BLBC (46/74, 62.2\%). In the current study, Raf-1 expression was confined to the invasive tumour components, with no staining in normal breast epithelial cells.

\section{Association of Raf-1 expression with clinicopathological data and patient prognosis}

The relationship between Raf-1 and other clinicopathological variables is summarised in Table 1. No significant correlation was observed in age, tumour size, histologic grade, BRCA1, and p53 protein expression between the positive and negative Raf-1 expression group of BLBC $(p>0.05)$. The proportion of histological grade 3 tumours was not significantly higher in the Raf-1-positive group than that of grade 2 tumours $(p>0.05)$. The percentage of tumours with higher Ki-67 expression ( $\geq 10 \%$ ) was also higher in the Raf-1-positive group than in the Raf-1-negative group $(73.5 \%$ vs. $26.5 \%, p<0.05)$. Since an increase in Raf-1 expression was associated with tumour metastasis in axillary lymph nodes, we examined which factor would contribute most significantly to a reduction in five-year disease-free survival (DFS). We performed Cox regression with multivariate analysis to identify the RR of reducing the five-year DFS. Table 2 shows that the following fac- 
Table 1. Expression of Raf-1 in basal-like carcinoma of the breast

\begin{tabular}{|c|c|c|c|c|c|}
\hline & $\begin{array}{c}\text { Case } \\
\text { No. }\end{array}$ & $\begin{array}{l}\text { Posi- } \\
\text { tive }\end{array}$ & $\begin{array}{l}\text { Nega- } \\
\text { tive }\end{array}$ & $\chi^{2}$ value & $P$ value \\
\hline \multicolumn{6}{|l|}{ Age } \\
\hline$\geq 50$ & 31 & 21 & 10 & & \\
\hline$<50$ & 43 & 25 & 18 & 0.357 & $>0.05$ \\
\hline \multicolumn{6}{|l|}{ Tumour size } \\
\hline $2 \leq$ and $<5 \mathrm{~cm}$ & 27 & 19 & 8 & & \\
\hline$<2 \mathrm{~cm}$ & 47 & 27 & 20 & 0.731 & $>0.05$ \\
\hline \multicolumn{6}{|l|}{ Histology grade } \\
\hline 1 & 0 & 0 & 0 & & \\
\hline ॥ & 13 & 9 & 4 & 0.07 & $>0.05$ \\
\hline III & 61 & 37 & 24 & & \\
\hline \multicolumn{6}{|l|}{ Lymph node } \\
\hline Metastasis & 52 & 37 & 15 & & \\
\hline Non-metastasis & 22 & 9 & 13 & 4.796 & $<0.05$ \\
\hline \multicolumn{6}{|l|}{ Ki-67 } \\
\hline$\geq 10 \%$ & 49 & 36 & 13 & & \\
\hline$<10 \%$ & 25 & 10 & 15 & 6.526 & $<0.05$ \\
\hline \multicolumn{6}{|l|}{ p53 } \\
\hline$\geq 10 \%$ & 41 & 29 & 12 & & \\
\hline$<10 \%$ & 33 & 17 & 16 & 2.112 & $>0.05$ \\
\hline \multicolumn{6}{|l|}{ BRCA1 } \\
\hline$\geq 10 \%$ & 23 & 15 & 8 & & \\
\hline$<10 \%$ & 51 & 31 & 20 & 0.011 & $>0.05$ \\
\hline
\end{tabular}

tors contribute significantly to a decrease in DFS: axillary lymph node metastasis and the tumours with higher Ki-67 expression and Raf-1 expression ( $\geq 10 \%$ positivity). Most interestingly, in addition to these traditional risk factors, higher Raf-1 expression was significantly associated with a decrease in five-year DFS (Fig. 2).

\section{Discussion}

The BLBC subtype accounts for $8-20 \%$ of breast cancer cases [13]. Recurrence of BLBC is frequently found within 1-3 years after treatment, and most patients with BLBC die within 5 years. Treating BLBC remains a challenge. Basal-like carcinoma of the breast is usually insensitive to endocrine therapy, and an effective targeted therapy has not yet been developed. At present, systemic chemotherapy is the most widely applied strategy, but the response to chemotherapy is unreliable [14]. This discloses that BLBC is a highly progressive disease, the prognosis of which is difficult to determine by clinical variables and molecular/ gene markers. Therefore, it is vital to understand the biological behaviours of this tumour to provide useful predictors for clinical outcomes and for guiding treatment. Currently, besides histological differentiation, lymph node status, clinical staging, and differentiation, other factors such as angiogenic markers [15], genetic markers [16], a member of the insulin-like growth factor-II (IGF-II) mRNA-binding protein family [17], cell cycle regulation [18], and PI3K pathway [19] have been reported to be indepen-
Table 2. Estimation of relative risk of reduction of disease-free survival in women with BLBC using multivariate analysis

\begin{tabular}{|c|c|c|c|}
\hline Biological factors & $\mathrm{RR}$ & $95 \% \mathrm{Cl}$ & $P$ value \\
\hline Tumour size ( $\geq 2$ vs. $<2 \mathrm{~cm}$ ) & 1.24 & $1.4-2.9$ & 0.16 \\
\hline $\begin{array}{l}\text { Lymph node (metastasis } \\
\text { vs. non-metastasis) }\end{array}$ & 1.96 & $1.1-3.5$ & 0.04 \\
\hline Age (<50 vs. $\geq 50$ years $)$ & 1.04 & $0.6-1.7$ & 0.68 \\
\hline $\begin{array}{l}\text { Ki-67 status }(\geq 10 \% \\
\text { vs. }<10 \% \text { expression })\end{array}$ & 2.14 & $1.0-3.3$ & 0.03 \\
\hline $\begin{array}{l}\text { p53 status ( } \geq 10 \% \\
\text { vs. }<10 \% \text { expression) }\end{array}$ & 1.19 & $0.5-1.6$ & 0.29 \\
\hline $\begin{array}{l}\text { BRCA1 status ( } \geq 10 \% \\
\text { vs. }<10 \% \text { expression) }\end{array}$ & 1.41 & $1.2-2.1$ & 0.19 \\
\hline $\begin{array}{l}\text { Raf- } 1 \text { status ( } \geq 10 \% \\
\text { vs. }<10 \% \text { expression) }\end{array}$ & 2.41 & $1.43-3.94$ & 0.02 \\
\hline
\end{tabular}

$R R$ - relative risk, $\mathrm{Cl}$ - confidence interval

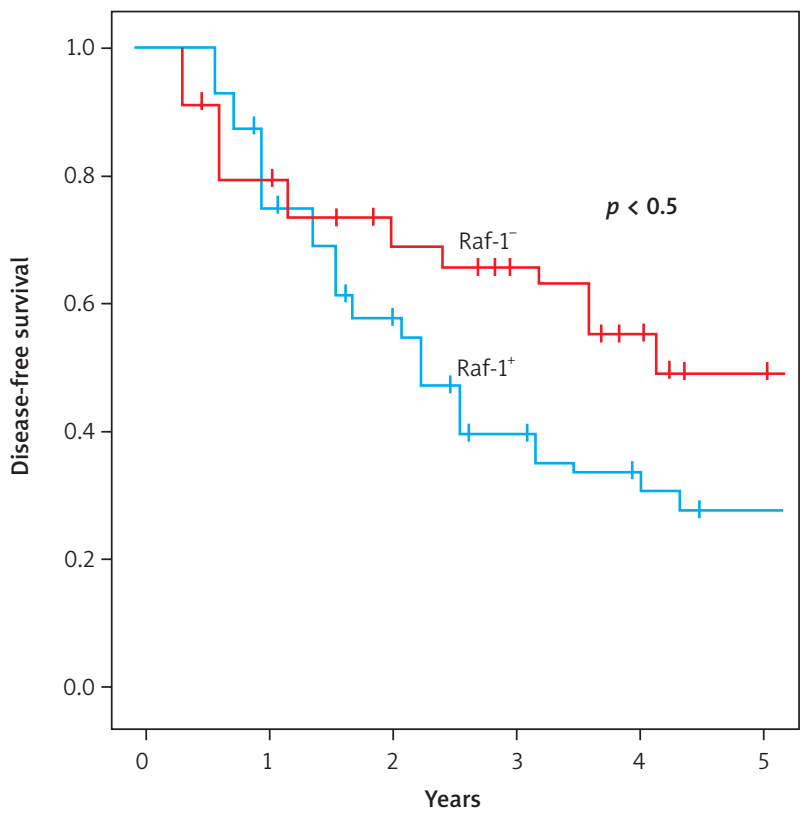

Fig. 2. Kaplan-Meier analysis of the probability of overall survival based on Raf-1 expressions in BLBC

dent prognostic markers for BLBC patients. However, some $B L B C$ are difficult to identify by their biological markers [20]. Consequently, additional prognostic biomarkers need to be identified that could aid stratification of breast carcinoma patients diagnosed with basal-like phenotype, and that could potentially explore novel treatment regimens.

The introduction of high-throughput technologies that survey hundreds to thousands of genes and their products in a single assay, coupled with powerful analytic tools, has opened new avenues for classifying breast cancer into biologically and clinically distinct groups based on gene expression patterns and DNA copy number alterations [21-25]. However, this technique is inhibitively expensive and complex, so it is not feasible to practice widely.

Raf-1 has been proven to contribute to the prognostic role in a variety of malignant tumours $[26,27]$. Indeed, high expression of Raf-1 has commonly been observed in solid tumours [9], including renal carcinoma, hepatocel- 
lular carcinoma, and NSCLC [28]. Increased expression of Raf-1 was also observed in cell lines and most malignant cells, suggesting that overexpression of Raf-1 protein may be related to the malignant transformation of cells [29]. Raf is considered as a unique gene of cancer, and various studies have established its important role in Ras/Raf/ MEK/ERK classic signalling pathway. It has been reported that activation of the Ras pathway predicts poor outcome on tamoxifen (endocrine therapy) in breast cancer, and identifies Raf-1 as a potential marker of resistance to oestrogen receptor-targeted therapy. In addition, it suggests that expression of Raf-1 could identify patients for whom tamoxifen alone is insufficient adjuvant systemic therapy, but for whom the addition of chemotherapy may be of benefit [30].

Immunohistochemical analyses from the recent $74 \mathrm{BLBC}$ patients suggest that Raf-1 protein was significantly highly expressed. However, there was no significant difference in Raf-1 expression between older and younger patients, bigger and smaller tumour size, grade 2 and grade 3 groups, positive and negative BRCA1, and p53 protein expression. The presence of inherited mutations in breast cancer susceptibility gene-1 (BRCA1) continues to be one of the best-defined overall risk factors for the development of breast cancer. Although the role of BRCA1 in hereditary breast cancers and the characteristics of tumours with germline mutation have been extensively studied, its role in sporadic tumours is still not well defined and there remains controversy regarding the significance of BRCA1 expression. There are several lines of evidence to suggest a link between BRCA1 deficiency and basal-like breast cancer [32]. Nevertheless, the current study did not find a link between BRCA1 expression and Raf-1 expression in BLBC. Such a pattern of expression may reflect differences in the specificity of antibodies used and the presence of different splice variant isoforms of BRCA1 [32]. Han et al. [33] demonstrated that Ki67 and p53 expression is significantly higher in triple-negative (TN) tumours compared to high-grade non-TN tumours, suggesting that Ki67 and p53 expression may play a role in worse prognosis of TN breast cancer. Furthermore, High expression of Ki67 (> 10\%), but not p53, shows better prognostic value. However, positive expression of Raf-1 was shown to be related to lymph node involvement and patient disease-free survival. These findings clearly demonstrate the prognostic value of Raf-1 for poor survival rate of BLBC patients when investigated and compared with clinical variables. Such findings also suggest that Raf-1 may be of predictive value for BLBC patients compared to TNM staging system and tumour differentiation, because most of BLBC are attributed to histological grade 3 or 2 .

The disadvantage of dividing BLBC patients into risk groups based on clinical and pathological description is the variation in interpreting these qualitative variables. As is already known, BLBC lacks ER, PR, and HER-2 expression, which are the most important prognostic factors. Therefore, the study is focused extensively on potential prognostic biomarkers, especially on BLBC-related protein expression profiles. Compared to genetic studies, which are expensive and not widely available, the protein expression studies by immunohistochemistry can be con- veniently carried out at any time because this technique is rapid, reproducible, and particularly low cost. In view of the current study, in which the positive expression of Raf-1 correlated well with poor survival rate of BLBC patients, the protein could be used as a predictor for clinical outcomes.

However, the sample size of this study was not large enough; it was a retrospective study by immunohistochemistry, so Raf-1 protein was not confirmed in BLBC by molecular biology. To understand how Raf-1 expression affects tumour cell fate, some in vitro experiments have also shown that Raf-1 could induce the proliferation of tumour cells [34], promote invasive ability, and arrest the cell cycle at the stage of $\mathrm{G}_{0} / \mathrm{G}_{1}$. Results from in vivo experiments are consistent with in vitro observations, indicating that downregulation of Raf-1 expression attenuates proliferation and induces apoptosis. Further preclinical and clinical studies are needed to verify the role of Raf- 1 in BLBC. The current study was impacted by the limited number of BLBC subtypes of breast carcinoma recruited to our record in a relatively small study population. In addition, the study relied solely on histological examination, and many interactions in vivo may need further study.

In conclusion, BLBC is among the greatest challenges given its high proliferation rate, rapid growth, early recurrence, and low overall survival. However, traditional clinicopathological prognostic markers may miss the inherence of breast carcinoma, leading to sub-optimal treatment and varied outcomes among patients with the same clinicopathological profiles. The current study found that increased expression of Raf-1 protein played an unfavourable role in patients' axillary lymph node metastasis and overall survival. The study implies that positive Raf-1 expression in $\mathrm{BLBC}$ could be considered as a new prognostic biomarker for poor survival of BLBC patients.

This study was supported by the National Natural Science Foundation of China (No. 30870975, No. 81272905).

The authors declare no conflict of interest.

\section{References}

1. Haupt B, Ro JY, Schwartz MR. Basal-like breast carcinoma: a phenotypically distinct entity. Arch Pathol Lab Med 2010; 134: 130-33.

2. Zhang H, Rakha EA, Ball GR, et al. The proteins FABP7 and OATP2 are associated with the basal phenotype and patient outcome in human breast cancer. Breast Cancer Res Treat 2010; 121: 41-51.

3. Ray PS, Wang J, Qu Y, et al. FOXC1 is a potential prognostic biomarker with functional significance in basal-like breast cancer. Cancer Res 2010; 70: 3870-6.

4. Liu Y, Jiang QY, Xin T, Cai L, Zhao CH. Clinical significance of basal-like breast cancer in Chinese women in Heilongjiang province. Asian Pac J Cancer Prev 2012; 13: 2735-8.

5. Valentin MD, da Silva SD, Privat M, Alaoui-Jamali M, Bignon YJ. Molecular insights on basal-like breast cancer. Breast Cancer Res Treat 2012; 134: 21-30.

6. Matos I, Dufloth R, Alvarenga M, Zeferino LC, Schmitt F. p63, cytokeratin 5, and P-cadherin: three molecular markers to distinguish basal phenotype in breast carcinomas. Virchows Arch 2005; 447: 688-94.

7. Niault TS, Baccarini M. Targets of Raf in tumorigenesis. Carcinogenesis 2010; 31: 1165-74. 
8. Casar B, Pinto A, Crespo P. Essential role of ERK dimers in the activation of cytoplasmic but not nuclear substrates by ERK-scaffold complexes. Mol Cell 2008; 31: 708-21.

9. Hagemann C, Gloger J, Anacker J, et al. RAF expression in human astrocytic tumors. Int J Mol Med 2009; 23: 17-31.

10. Bauer KR, Brown M, Cress RD, Parise CA, Caggiano V. Descriptive analysis of estrogen receptor (ER)-negative, progesterone receptor (PR)-negative, and HER2-negative invasive breast cancer, the socalled triple-negative phenotype: A population based study from the California cancer Registry. Cancer 2007; 109: 1721-8.

11. Livasy CA, Karaca G, Nanda R, Tretiakova MS, Olopade OI, Moore DT, Perou CM. Phenotypic evaluation of the basal-like subtype of invasive breast carcinoma. Mod Pathol 2006; 19: 264-71.

12. Cheang MC, Voduc D, Bajdik C, Leung S, McKinney S, Chia SK, Perou CM, Nielsen TO. Basal-like breast cancer defined by five biomarkers has superior prognostic value than triple-negative phenotype. Clin Cancer Res 2008; 14: 1368-76.

13. Dey N, Smith BR, Leyland-Jones B. Targeting basal-like breast cancers. Curr Drug Targets 2012; 13: 1510-24.

14. Pazaiti A, Fentiman IS. Basal phenotype breast cancer: implica tions for treatment and prognosis. Womens Health (Lond Engl) 2011; 7: 181-202.

15. Horiguchi H, Yamagata S, Rong Qian Z, Kagawa S, Sakashita N. Thrombospondin-1 is highly expressed in desmoplastic components of invasive ductal carcinoma of the breast and associated with lymph node metastasis. J Med Invest 2013; 60: 91-6.

16. Mulligan AM, Pinnaduwage D, Bane AL, Bull SB, O'Malley FP, Andrulis IL. CK8/18 expression, the basal phenotype, and family history in identifying BRCA1-associated breast cancer in the Ontario site of the breast cancer family registry. Cancer 2011; 117: 1350-9.

17. Walter O, Prasad M, Lu S, Quinlan RM, Edmiston KL, Khan A. IMP3 is a novel biomarker for triple negative invasive mammary carci noma associated with a more aggressive phenotype. Hum Pathol 2009; 40: 1528-33.

18. Thorner AR, Hoadley KA, Parker JS, Winkel S, Millikan RC, Perou CM. In vitro and in vivo analysis of B-Myb in basal-like breast cancer. Oncogene 2009; 28: 742-51.

19. López-Knowles E, O’Toole SA, McNeil CM, et al. PI3K pathway activation in breast cancer is associated with the basal-like phenotype and cancer-specific mortality. Int J Cancer 2010; 126: 1121-31.

20. Li D, Xiao X, Yang W, Shui R, Tu X, Lu H, Shi D. Secretory breast carcinoma: a clinicopathological and immunophenotypic study of 15 cases with a review of the literature. Mod Pathol 2012; 25: 567-75.

21. Sotiriou C, Pusztai L. Gene-expression signatures in breast cancer. N Engl J Med 2009; 360: 790-800

22. Eroles P, Bosch A, Pérez-Fidalgo JA, Lluch A. Molecular biology in breast cancer: intrinsic subtypes and signaling pathways. Cancer Treat Rev 2012; 38: 698-707.

23. Fan C, Oh DS, Wessels L, Weigelt B, Nuyten DS, Nobel AB, van't Veer LJ, Perou CM. Concordance among gene expression-based predictors for breast cancer. N Engl J Med 2006; 355: 560-9.

24. Haibe-Kains B, Desmedt C, Piette F, et al. Comparison of prognostic gene expression signatures for breast cancer. BMC Genomics 2008; 9: 394.

25. Sotiriou C, Wirapati P, Loi S, et al. Gene expression profiling in breast cancer: understanding the molecular basis of histologic grade to improve prognosis. J Natl Cancer Inst 2006; 98: 262-72.

26. Cekanova M, Majidy M, Masi T, Al-Wadei HA, Schuller HM. Overexpressed Raf-1 and phosphorylated cyclic adenosine 3'-5'-monophosphatate response element-binding protein are early markers for lung adenocarcinoma. Cancer 2007; 109: 1164-73.

27. Mukherjee R, Bartlett JM, Krishna NS, Underwood MA, Edwards Raf-1 expression may influence progression to androgen insensitive prostate cancer. Prostate 2005; 64: 101-7.

28. Chen L, Shi Y, Jiang CY, Wei LX, Wang YL, Dai GH. Expression and prognostic role of pan-Ras, Raf-1, pMEK1 and pERK1/2 in patients with hepatocellular carcinoma. EJSO 2011; 37: 513-20.

29. Niault TS, Baccarini M. Targets of Raf in tumorigenesis. Carcinogenesis 2010; 31: 1165-74.

30. McGlynn LM, Kirkegaard T, Edwards J, Tovey S, Cameron D, Twelves C, Bartlett JM, Cooke TG. Ras/Raf-1/MAPK pathway mediates response to tamoxifen but not chemotherapy in breast cancer patients. Clin Cancer Res 2009; 15: 1487-95.

31. Turner NC, Reis-Filho JS. Basal-like breast cancer and the BRCA1 phenotype. Oncogene 2006; 25: 5846-53.

32. Rakha EA, El-Sheikh SE, Kandil MA, El-Sayed ME, Green AR, Ellis IO. Expression of BRCA1 protein in breast cancer and its prognostic significance. Hum Pathol 2008; 39: 857-65.

33. Han JS, Cao D, Molberg KH, Sarode VR, Rao R, Sutton LM, Peng Y. Hormone receptor status rather than HER2 status is significantly associated with increased Ki-67 and p53 expression in triple-negative breast carcinomas, and high expression of Ki-67 but not p53 is significantly associated with axillary nodal metastasis in triple-negative and high-grade non-triple-negative breast carcinomas. Am J Clin Pathol 2011; 135: 230-7.

34. Leontovich AA, Zhang S, Quatraro C, et al. Raf-1 oncogenic signaling is linked to activation of mesenchymal to epithelial transition pathway in metastatic breast cancer cells. Int J Oncol 2012; 40: 1858-64.

\section{Address for correspondence}

\section{Prof. Weiqiang Zheng}

Department of Pathology

Changhai Hospital, Second Military Medical University

Shanghai, China

e-mail: zheng6947@126.com

Submitted: 17.11 .2013

Accepted: $\quad 3.02 .2014$ 